\title{
Archéopages
}

Archéopages

Archéologie et société

Hors-série 1 | 2008

Constructions de l'archéologie

\section{Durabilité et archéologie environnementale}

\author{
Sander van der Leeuw
}

\section{(2) OpenEdition}

Journals

Édition électronique

URL : https://journals.openedition.org/archeopages/870

DOI : 10.4000/archeopages. 870

ISSN : 2269-9872

Éditeur

INRAP - Institut national de recherches archéologiques préventives

Édition imprimée

Date de publication : 1 février 2008

Pagination : $97-102$

ISSN : 1622-8545

Référence électronique

Sander van der Leeuw, «Durabilité et archéologie environnementale », Archéopages [En ligne], Horssérie 1 | 2008, mis en ligne le 01 février 2008, consulté le 24 février 2023. URL : http://

journals.openedition.org/archeopages/870 ; DOI : https://doi.org/10.4000/archeopages.870

Tous droits réservés 
la main-d'œuvre des chantiers archéologiques n'a été que rarement cantonnée au rôle d'exécutant ou de musculature harnachée au service de la science. Sans doute, cette main-d'œuvre a soulevé divers enjeux de rentabilité et de productivité, sans parler de gestion humaine et de contrôle. Boucher de Perthes, précurseur qu'il était, n’a pu échapper à la supercherie de certains de ses ouvriers, qui exhumaient opportunément les faux bifaces qu'ils avaient taillés la veille. D'autres ont mieux su se prémunir: c'est littéralement la jumelle collée à l'œil que l'égyptologue Flinders Petrie arpentait son tell pharaonique pour mieux surveiller les agissements de ses fellahin. De même, il n'a pas échappé à Mortimer Wheeler que sa méthode de fouille par «boxes» de 5 mètres sur 5 permettait de mieux contrôler les fouilleurs, qu'ils appartinssent aux classes laborieuses anglaises ou qu'ils fussent des sujets colonisés.

Mais, en même temps, maints ouvriers se sont investis dans leur gagne-pain en tant qu'êtres humains, avec motivation et fascination. Déjà Boucher de Perthes, grand seigneur, reconnaissait que la sagacité et la persévérance de certains de ses terrassiers leur donnaient lieu de croire qu'ils étaient des savants: "Leur satisfaction était au comble quand, sur, l'étiquette [des pièces qu'ils avaient trouvées] ils apercevaient leur nom. Il en est qui ont refusé le prix d'un morceau en me disant: $<$ Je voudrais seulement qu'on parlât de moi dans le livre `» (Meunier 1875, p. 37). Au-delà donc de l'aspect financier, des ouvriers curieux et dignes de confiance se distinguèrent rapidement auprès des préhistoriens anglais Evans et Prestwich, puis chez le général Pitt Rivers, qui forma sur ses sites plusieurs contremaitres spécialisés. En Orient, c'est en se pliant aux mours locales que Petrie érigea les villageois de Quft en véritable guilde de fouilleurs professionnels. En Afrique subsaharienne, le préhistorien et ethnographe des Mau-Mau, Louis Leakey, sut distinguer, parmi les «boys» qu'il recrutait localement, les plus talentueux, notamment un certain Juma, son meilleur trouveur de fossiles.

Même s'ils ne sortent que peu fréquemment de l'anonymat et reçoivent plus rarement encore le crédit qui leur est dû (et encore, on le voit ici même, plutôt dans les documents et les photos d'archives que dans les publications), ces ouvrierssavants révèlent par leur apparente transgression quelques-unes des spécificités profondes de l'archéologie. À la différence de bien d'autres activités, économiques, techniques mais aussi scientifiques, on peut voir en archéologie une certaine cohérence entre la production du savoir et son contenu. Déjà l'engagement physique du présent, le dispositif d'exhumation, est en quelque sorte en résonance avec la matérialité du passé et de ses vestiges enfouis. De plus, cet engagement se concrétise aisément dans le temps et dans l'espace: sans nier bien évidemment l'importance du laboratoire ni de la bibliothèque, c'est durant les quelques semaines de la campagne de fouille, sur le terrain, que se concentrent les énergies et les consciences. Dans la foulée, savants et ouvriers se côtoient et se salissent en dirigeant leurs efforts communs, intellectuels et manuels, sur les hommes du passé. Et, alors même que se maintient le rythme de travail, que les pelletées s'accumulent et que les brouettes se déchargent sans encombre, il y a dans cet engagement laborieux avec les vestiges du passé humain - des habitations, des inhumations, des restes d'activités quotidiennes, des objets de parure et de prestige, des symboles et des signes (comme, par exemple, des mains à doigts coupés imprimées sur des parois de grottes ornées) - une incitation à la réflexion et au rêve.

Cette vocation est encore plus pertinente dans l'archéologie moderne d'aujourd'hui. Cette archéologie qui s'inspire des principes scientifiques et patrimoniaux de la convention de Malte et qui cherche à contrer l'érosion du passé par une politique de développement durable. Cette archéologie qui sait s'adapter aux échelles et aux échéances de l'aménagement du territoire et remplacer, quand l'occasion le demande, le pénible maniement d'une pelle par le godet lisse d'un engin mécanique. Autant du point de vue interne - celui du métier, de la formation, de la responsabilisation que de celui externe - au niveau des institutions, des opérations, des partenariats -, cette fusion du travail manuel et intellectuel ne fait que confirmer tout l'intérêt que représente l'archéologie, entre science et passion... un antidote à l'aliénation et au désenchantement.

Meunier V. 1875 : Les Ancêtres d'Adam. Histoire de l'homme fossile, Paris, Rothschild éditeur.

\section{Durabilité et archéologie environnementale}

Sander van der Leeuw

Arizona State University

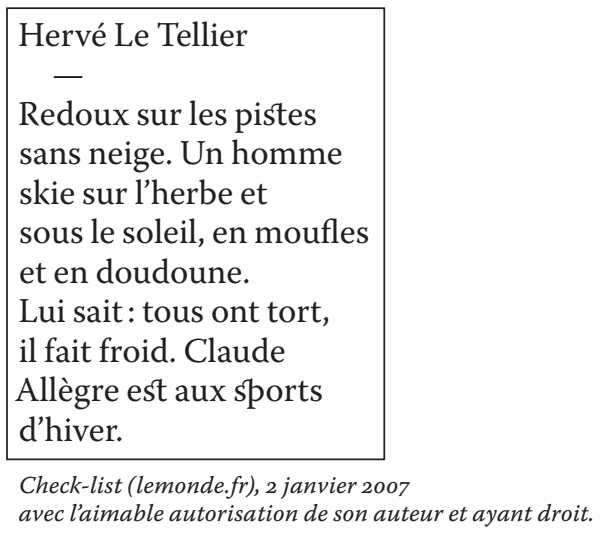

L'environnement, au risque de déplaire... Que cela plaise ou non, force est de constater que notre environnement est en train de changer. L'ouverture, dans le Grand Nord canadien, d'une route maritime reliant l'Europe à l'ExtrêmeOrient n'en est qu'une manifestation parmi 


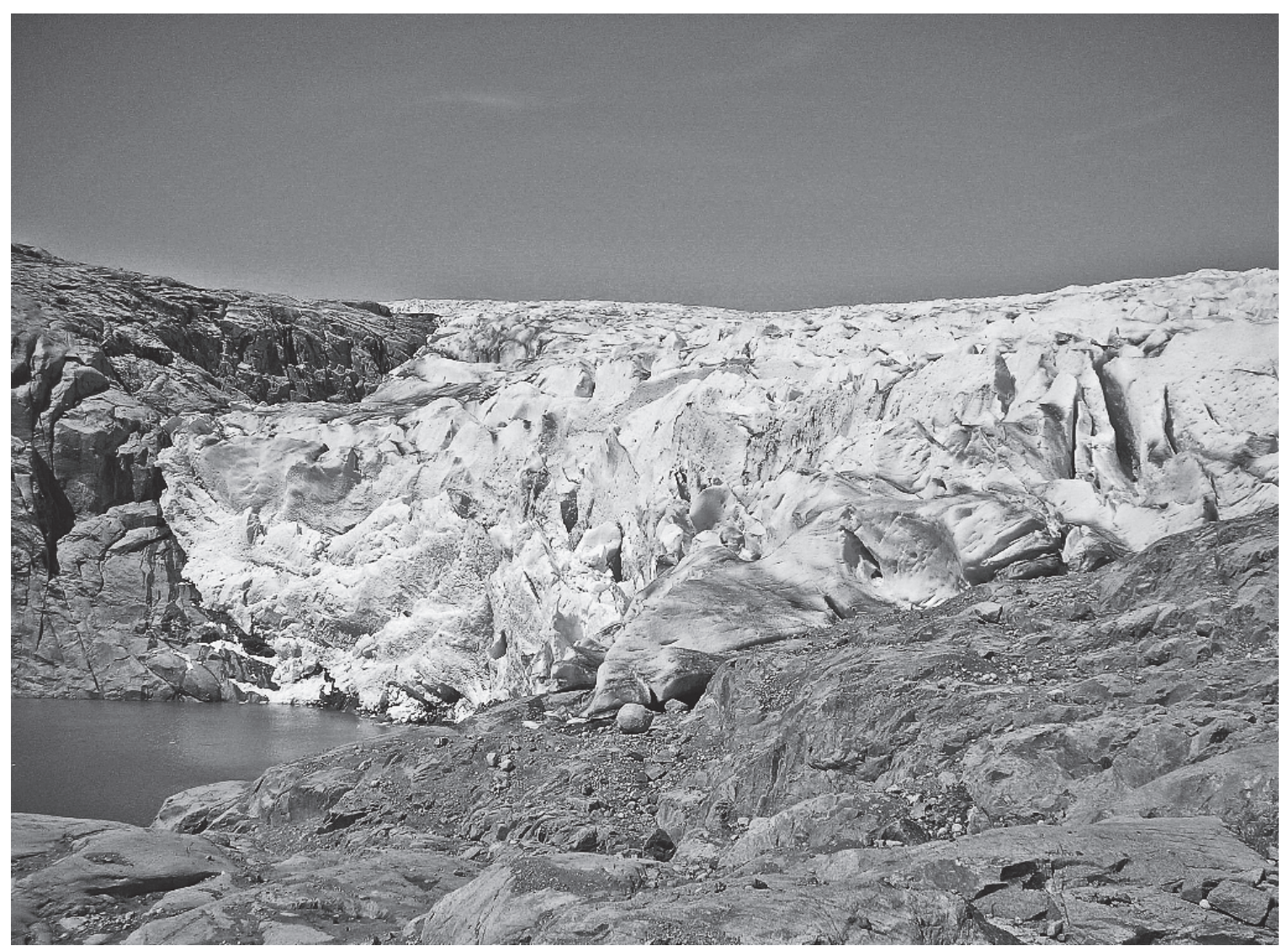

[Fig. 1] Retrait d'un glacier, près de Narsaq, Groenland, 28 juin 2007. 
d'autres, que cela soit la fonte des neiges éternelles du Kilimanjaro, du Popocatépetl ou du Fuji-Yama, ou encore le recul de plus en plus rapide des glaciers du Groenland [Fig.1]. C'est ainsi que le glacier d'Ilulissat - le plus grand producteur d'icebergs de l'hémisphère nord - a reculé de 1okilomètres entre 2001 et 2003 , soit presque autant qu'en un siècle; il libère actuellement 3o millions de tonnes de glace par jour, soit une augmentation de cent pour cent du volume quotidiennement déversé dans la mer au cours des années 1990.

Durant les vingt dernières années au début desquelles ces manifestations ont été remarquées-, scientifiques et politiciens n'ont jamais su s'accorder sur la cause du phénomène: cela est-il - oui ou non - dû à l'action de l'homme? La bataille fait toujours rage, même si la très grande majorité de la communauté scientifique - telle qu'elle est représentée par le Groupe d'experts intergouvernemental sur l'évolution du climat (GIEC), l'Académie des sciences des États-Unis, le Programme GéosphèreBiosphère ou encore l'Organisation météorologique mondiale - affirme aujourd'hui que les gaz à effet de serre en sont la cause principale. En France, en dépit de Claude Allègre (2007) qui a récemment rejoint les «négationnistes» conduits par le très véhément Bjørn Lomborg (2003, 2007), les scientifiques prennent ce problème très au sérieux. La cause du changement climatique n'est pourtant pas la question primordiale; pas plus que ne l'est la querelle sur le nombre exact de degrés de la future augmentation de la température dans les différentes régions du globe. La question essentielle est autre: comment l'humanité telle qu'elle est au XXI ${ }^{e}$ siècle-va-t-elle faire face à de tels bouleversements?

Les preuves viennent de partout ${ }^{\mathbf{1}}$ sur les conséquences dramatiques que pourraient avoir ces changements; elles impliquent une montée considérable du niveau de la mer, un brutal changement des courants océaniques qui régulent le climat de l'hémisphère Nord, un accroissement de la fréquence des cyclones, des ouragans et des tornades, des sécheresses dans des lieux inhabituels avec des pénuries de nourriture, sans parler des bouleversements sociaux causés par les migrations, les famines

1 Voir à cet égard

les rapports du GIEC

(1990, 1995, 2001

et 2007); ces rapports

et beaucoup d'autres

sont disponibles

sur le site Internet

du Intergovernmental

Panel on Climate Change

ou IPCC: http://www.

ipcc.ch/ipccreports/

index.htm; voir

également le Rapport

Stern (2006),

le Rapport 2007-2036

du Development,

Concepts and Doctrine

Centre du ministère

britannique de

la Défense (2007), et les publications

de réflexion ou

think-tanks en sciences

sociales. et l'interruption brutale des flux de ressources sur lesquels nos sociétés se sont construites et se reproduisent actuellement.

Quelle nature voulons-nous? Comme le font correctement remarquer de nombreux archéologues, l'humanité a déjà traversé ce type de crise et y a survécu (Thiébault 2008). Cependant, depuis le Néolithique, l'interaction entre les êtres humains et leur environnement a fondamentalement changé. Les populations humaines ne prélèvent plus simplement ce que la nature leur offre directement. Elles ont désormais - partout et de manière substantielle«investi» leur environnement pour élaborer un mode de vie totalement dépendant de ces investissements, et non plus du seul environnement. Ces engagements ne concernent pas seulement l'environnement matériel et construit, mais également nos institutions, notre démographie, nos gestes quotidiens, nos valeurs, nos catégorisations et

les représentations mentales avec lesquelles nous concevons et construisons notre univers et nos relations avec autrui, etc.

Les investissements matériels sont, à eux seuls, si conséquents qu'il serait impossible de mettre en ouvre - avant qu'il ne soit trop tard et avec une équité à l'échelle mondiale - les moyens nécessaires pour contrer les conséquences du changement climatique; au demeurant, plus on attend, plus les risques et les coûts augmentent (Costanza et al. 1997; Stern 2006). Mais il sera encore plus difficile d'effectuer les nécessaires changements mentaux, culturels et sociaux qui, seuls, nous permettront de relever le défi qui nous est lancé. Au demeurant, nous n'avons même pas commencé à penser de manière proactive aux directions à prendre et à poser la question primordiale: Quelle nature voulons-nous? (Levêque, van der Leeuw 2003). Nous n'avons su que ré-agir contre ce que nous ne voulons pas, à savoir la pollution, la pénurie d'énergie, le changement climatique, etc.

Nos actes et nos décisions sont actuellement infléchis par des modèles et des scénarios scientifiques (proposés par des organisations comme l'OCDE, la Banque mondiale et les différents gouvernements), ainsi que par des projections des conséquences du changement climatique et environnemental. Cette procédure est pourtant problématique et dangereuse: ces modèles sont en effet basés sur des données numériques (météorologiques, sociales, économiques, etc.) qui ne sont disponibles que depuis une cinquantaine d'années (tout au plus, pour certaines d'entre elles, un ou deux siècles). En conséquence, non seulement les processus non observables à une telle échelle temporelle sont exclus (parce que leur rythme est plus lent), mais le sont également de nombreux états de système qui peuvent avoir existé dans un passé lointain. Par ailleurs, les échantillons reflètent une Terre profondément affectée par des déséquilibres que l'on juge actuellement 
dangereux; c'est un peu comme si un médecin regardait un malade sans avoir jamais vu un homme en bonne santé. Les modèles et les scénarios ne prennent donc en compte ni le changement du changement ni les différentes manières dont les processus naturels et sociaux peuvent avoir évolué sur le très long terme.

L'archéologie est l'une des rares disciplines (sinon la seule) à même de pouvoir contribuer à la compréhension des anciennes dynamiques entre le climat, les environnements naturels terrestres et les êtres humains. Elle dispose en effet d'enregistrements qui remontent à des centaines de milliers d'années; pour le dernier millénaire, les informations sont même extrêmement détaillées, issues d'un travail scientifique rigoureux et référencées dans l'espace. Quelle que soit la position des archéologues dans le débat, ils doivent aux générations futures de faire de leur mieux pour enquêter sur les systèmes socio-naturels passés, et exposer leurs conclusions le plus impartialement possible. Cette tâche est en train de devenir urgente car, selon certains, nous n'avons que jusqu'au milieu du millénaire pour concevoir des solutions et les mettre en œuvre.

\section{La situation et le devoir de l'archéologie environnementale française. Au cours des vingt} dernières années, la France a promu une petite mais excellente communauté d'archéologues environnementalistes. Cette communauté compte un nombre important de réalisations dans les domaines du paléoclimat, de l'archéozoologie et des végétaux anciens. Elle s'est très tôt investie dans les analyses isotopiques et la génétique des anciennes populations humaines, animales et végétales. L'un de ses grands succès est l'utilisation novatrice de la micromorphologie des sols pour reconstituer les paysages; un autre est le développement d'une base de données opérationnelle. Reste que l'archéologie environnementale n'est toujours pas inscrite dans le protocole normal des prospections, fouilles et programmes régionaux; l'environnement reste, en effet, l'enfant pauvre des budgets.

La mobilisation a, par ailleurs, été insuffisante pour montrer que l'histoire longue de l'environnement est une donnée constitutive de la compréhension des dynamiques socioenvironnementales du présent et un élément capital du débat sur la durabilité. L'ordre du jour de la recherche en archéologie environnementale continue à n'être orienté que par des questions académiques, alors qu'elle peut et doit contribuer à l'avenir des futures générations. Espérons qu'il n'est pas déjà trop tard pour se lancer dans une telle entreprise.

Les raisons de ces limitations sont multiples. En premier lieu, l'archéologie environnementale française est jeune. Dans les pays d'Europe du Nord, elle a débuté au Xıx ${ }^{\mathrm{e}}$ ou au tout début du $\mathrm{xx}^{\mathrm{e}}$ siècle. En France, elle ne s'est développée qu'à partir des années 1980; le temps a donc été trop court pour que se crée une véritable culture environnementaliste au sein de la communauté archéologique. En deuxième lieu (et en conséquence de sa jeunesse), la communauté qui anime la discipline est relativement restreinte. Cette dernière n'est enseignée que dans très peu d'universités et n'a produit qu'un nombre limité de doctorats. Les protagonistes n'ont donc pas encore suffisamment conquis de positions d'autorité dans le monde académique pour assurer l'avenir de leur communauté.

Enfin, l'archéologie environnementale a été confrontée au bouleversement de l'archéologie préventive, bien avant de s'être établie en tant que telle.

Au cours des années 1960 et jusqu'à la fin des années 1970, l'archéologie fut, dans la plupart des pays européens, essentiellement une discipline scientifique pratiquée dans le cadre d'universités et d'organismes de recherche. Elle était donc le fait de questionnements purement scientifiques. On croyait alors à la «bonne» science et à ses bénéfices pour la société. Durant cette période, les techniques et les méthodes scientifiques ont connu de grandes avancées qui ont été reprises par les archéologues. Mais, en France, l'archéologie environnementale n'était pas au rendez-vous.

Le climat social et intellectuel commença à changer au cours des années 1980. Les «trente glorieuses », qui avaient succédé à la Seconde Guerre mondiale, touchaient à leur fin, les fonds devenaient plus rares et l'archéologie scientifique des universités, à l'image d'autres disciplines mal perçues ou financièrement non rentables, commença à en pâtir. C'est alors que, partout dans le monde, l'archéologie se protégea en avançant l'argument de la sauvegarde patrimoniale ou celui de la préservation de vestiges exceptionnels non reproductibles pouvant attirer les touristes. Ce n'est qu'au Xxi ${ }^{\mathrm{e}}$ siècle, et après presque tous les pays développés, que la France (première destination touristique du monde, détentrice d'un riche patrimoine culturel et d'un ministère de la Culture) a réglementé cette dynamique. Le centre de gravité de l'archéologie s'était alors déplacé de l'archéologie académique et savante vers l'archéologie préventive. Le projet de réforme du CNRS, qui envisage de donner la responsabilité de la recherche archéologique aux universités lesquelles n'ont pourtant jamais assuré ce type d'administration-, va probablement accélérer cette tendance.

Les conséquences de ce qui est train de se passer ont des répercussions plus profondes. Avoir substitué la sauvegarde patrimoniale aux problématiques archéologiques a sérieusement érodé la crédibilité de la discipline dans le concert des sciences. Par ailleurs, la préservation patrimoniale - prise dans la globalisation d'un monde organisé en États-nations - conduit inévitablement à des démarches (construction identitaire, conflits politiques, etc.) qui n'ont rien de scientifique. Le choix archéologique - 
pourquoi fouiller un site plutôt qu'un autre?n'est désormais plus sous-tendu par des questionnements scientifiques mais par des sous-entendus idéologiques: la perception de ce qui nous paraît culturellement important ou la possibilité de retombées financières, voire le renforcement du statut de certains segments de la population...

L'ancien système n'était certes pas sans défaut, mais les dérives du système actuel sont réelles. $\mathrm{Au}$ vu de ce qui s'est passé dans les pays qui ont privilégié l'archéologie préventive (la gestion des ressources culturelles, comme on dit dans les pays anglo-américains), il semble une fois la machine lancée- que ce soient le maintien de l'institution et la préservation des emplois qui prévaillent; la majorité des décisions sont alors déterminées par des raisons extérieures aux questionnements scientifiques.

\section{L'Inrap et l'archéologie environnementale.} Ce n'est pas le moindre des mérites de l'Inrap, appuyé par un petit cercle de personnes, que d'avoir su faire en sorte que la France ait désormais une structure organisationnelle nettement plus cohérente que celle de nombreux pays développés où ce sont des compagnies privées qui effectuent la majorité des travaux archéologiques. Cependant, l'Inrap pourrait bientôt ne plus être l'institution dominante de l'archéologie française, mais en devenir le seul refuge. Si la réforme annoncée de la recherche sort des limbes où elle semble s'élaborer, c'est probablement à l'Inrap que l'archéologie française trouvera les moyens de son maintien dans la communauté internationale - celle qui tente de trouver les informations qui décideront du futur de nos sociétés. Depuis sa création, l'Inrap a agi en faveur du renforcement de l'archéologie environnementale. Espérons que sa politique continuera d'être aussi avisée et efficace à l'égard d'une cause devenue fondamentale.

Vers quelle évolution? En premier lieu, il est nécessaire que l'Inrap et les archéologues déplacent leurs centres d'intérêt. Ils doivent s'engager ouvertement et vigoureusement dans le débat actuel en s'appuyant sur toutes les possibilités (financières ou autres).

Il ne faut certes pas éliminer les tours d'ivoire de la recherche fondamentale, tout en sachant qu'elles ne sont pas l'urgence. Même si la recherche «indépendante» et «objective» répond aux questions posées, on sait que ces dernières sont subjectives et inscrites dans des contextes politiques et culturels particuliers.

Les archéologues doivent également surmonter leur manque de confiance dans leurs résultats, qui ne sont pas moins fondés que ceux des autres disciplines. Nous sommes des experts à part entière, et nous sommes bons! Ne pas être pris en compte dans le nouveau débat constituerait une erreur coûteuse... Pourtant, les archéologues environnementalistes ne furent guère conviés $\mathrm{au}$ «Grenelle de l'environnement» récemment orchestré par le gouvernement français.

En deuxième lieu, la communauté de l'archéologie environnementale doit aller plus loin dans ses collaborations interdisciplinaires, avec les sciences sociales en particulier. Le débat sur le réchauffement s'est récemment déplacé: là où l'on ne voyait que des solutions et des problèmes environnementaux, on comprend aujourd'hui que ce sont les sociétés humaines qui définissent leur environnement, leurs problèmes environnementaux et les solutions à y apporter. De fait, les difficultés sont essentiellement d'ordre social et non environnemental; en effet, quoi qu'il advienne, l'environnement continuera, quant à lui, d'exister. Dans l'«anthropocène» (Crutzen, Stoermer 2000) où nous vivons depuis plusieurs siècles, l'espèce humaine a eu un tel impact sur l'environnement non humain qu'on ne peut plus les distinguer l'une de l'autre. Le système «socio-naturel» est déterminé par des dynamiques sociales qui entrent en action bien avant les premiers signes critiques. Par ailleurs, internationalement (Direction de la recherche de l'Union européenne, Conseil européen de la recherche, Fondation européenne pour la science, etc.) et nationalement (ANR, CNRS, régions, etc.), ce sont les projets interdisciplinaires qui seront prochainement la principale source de financement de l'archéologie environnementale.

En troisième lieu, la communauté et ses institutions doivent réorganiser la formation. Les dernières années ont montré (en France, tout particulièrement) à quel point une institution ou une politique de recherche dépendait de quelques individus seulement. L'archéologie environnementale a besoin de plusieurs écoles doctorales; cette multiplication serait d'ailleurs compatible avec le projet - s'il voit jamais le jourd'une autonomie des universités.

En quatrième lieu, les profils de carrière de «terrain» doivent être développés. On devrait pouvoir profiter de la création d'un Département des sciences environnementales et du développement durable, au CnRs. Ce dernier a longtemps aidé à créer, à promouvoir et à stimuler l'archéologie environnementale. Dans la conjoncture actuelle, il serait ironiquement amer de voir disparaitre ce type d'initiative. Cependant, une grande part des responsabilités incombent aujourd'hui à l'Inrap, en tant que principal employeur d'archéologues et principal fournisseur de données environnementales. Des postes doivent être créés à différents niveaux pour satisfaire les besoins de la recherche archéologique, mais aussi et surtout pour répondre aux questions du public. Le problème actuel est que trop peu d'individus doivent simultanément répondre à des types trop différents de question.

En cinquième lieu, l'archéologie environnementale doit créer les bases de sa propre institutionnalisation, et poursuivre activement la mise en place de ses membres les plus actifs et reconnus à des postes-clés (Institut universitaire 
de France, Collège de France, Académie des sciences, etc.), afin de créer le parapluie ou le parasol sous lequel elle pourra prospérer.

Enfin, la communauté doit comprendre que la recherche n'est plus le fait d'individus ou d'équipes singulières, mais d'un ensemble de scientifiques issus de différentes disciplines. Les archéologues environnementalistes français sont internationalement beaucoup moins présents dans la recherche environnementale que ceux des autres communautés linguistiques. Peut-être du fait que le français n'est plus aujourd'hui que la douzième langue parlée dans le monde? Pourtant, toutes les nations ont en main un atout majeur: l'intérêt du public pour les questions environnementales et la durabilité. Académies des sciences, gouvernements et - aujourd'hui, beaucoup plus important-médias et citoyens du monde se sentent concernés, même si quelques «prophètes de la fin du monde» prédisent une rapide défection. De fait, et aussi longtemps que les désastres «naturels» dus au réchauffement de la planète continueront d'augmenter en nombre et en puissance, les sociétés ne cesseront de placer ces questions au cour de leurs priorités.

En conclusion... L'archéologie environnementale est, en France, à un tournant critique. Si la communauté se rassemble, change ses comportements et travaille à son renforcement, elle sera capable de profiter du changement climatique. Pour ce faire, il existe un certain nombre d'atouts à la fois médiatiques, grand public et politiques, aussi bien au CNRS qu'à l'Inrap. Avec quelques «leaders», quelques efforts et une bonne stratégie, on peut aboutir à beaucoup. Si cela ne se produit pas, la discipline est menacée d'atrophie.

Allègre C. 2007: Ma vérité sur la planète, Paris, Plon.

Costanza R., d'Arge R., De Groot R., Farberk S., Grasso M. Hannon B., Limburgi K., Naeem S., O’Neill R.V., Paruelo J., Raskin R.G., SutTonKK P., VAN DEN Belt M. 1997:

"The Value of the World's Ecosystem Services and natural Capital», Nature, 387 , p. 253-26o.

Crutzen P. J., Stoermer E. F. 2000 : «The Antropocène», IGBP Newsletter, 41, p.17-18.

Development, Concepts and Doctrine Centre 2007: Global Strategic Trends Program 2007-2036, Londres, UK Department of Defense; www.mod.uk/DefenceInternet/AboutDefence/WhatWeDo/ DoctrineOperationsandDiplomacy/DCDC/ DcdcStrategicTrendsPublications.htm

Groupe d'experts intergouvernemental sur l'évolution du climat 1990: Intergovernmental Panel on Climate Change (IPCC) First Assessment Report. Cambridge, Cambridge University Press.

1995 : Deuxième Rapport d'évaluation du GIEC: Changements climatiques 1995 ; http://www.ipcc.ch/languages/french.htm\#21

2001: Troisième Rapport d'évaluation du GIEC. Bilan 2001 des changements climatiques; http://www.ipcc.ch/languages/french. htm\#21

2007: Climate Change 2007, the Fourth IPCC Assessment Report; http://www.ipcc.ch/ipccreports/index.htm

LÉVÊQUE CHR., VAN DER LEEUW S. E. (dir.) 2003: Quelles natures voulons nous? Pour une approche socio-écologique du champ de l'environnement, Paris, Elsevier.

Lomborg B. 2004: L'Écologiste sceptique. Le véritable état de la planète, Paris, Éd. du Cherche-Midi.

LOMBORG B. 2007: Cool It - The Skeptical Environmentalist's Guide to Global Warming, New York, Knopf.

STERN Sir N. 2006: Review Report on the Economics of Climate Change, Londres, HM Department of the Treasury; www.hm-treasury.gov. uk/independent_reviews/
À paraître,

ThiéBault S. «L'homme et le climat: l'apport de l'archéologie environnementale à la connaissance des relations homme-climat», in J.-P. Demoule (dir), L’Avenir du passé, modernité de l'archéologie, actes du colloque international de l'Institut national de recherches archéologiques préventives et du Centre Pompidou, 23-24 novembre 2006, Paris; http://www.centrepompidou.fr/Pompidou/WebTV. nsf/o/D4B6914D4CA520E7 C1256F3Aoo5DD9D5?OpenDocument \&session $\mathrm{M}=5.2 \& \mathrm{~L}=1$ 